\title{
Medical Image of the Week: Atherosclerotic Aneurysm of Great Vessels
}

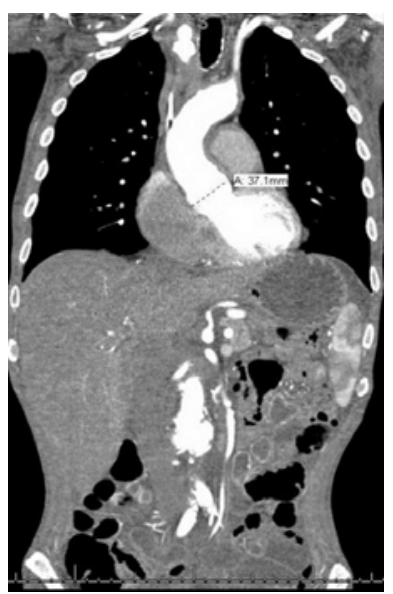

Figure 1.Unsual appearance of the aortic root with irregular contours and a $41 \times 37 \mathrm{~mm}$ dilatation at the level of the sinuses of Valsalva.

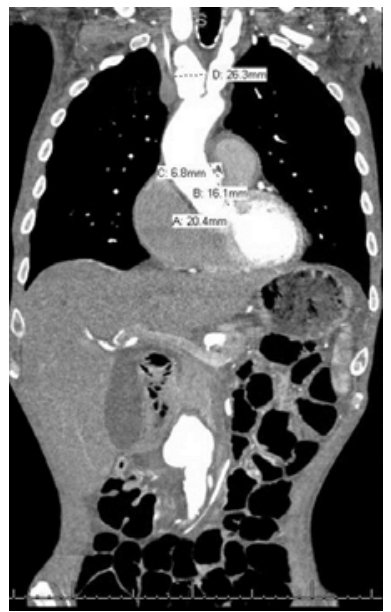

Figure 2. Saccular outpouchings of the intrathoracic great vessels.

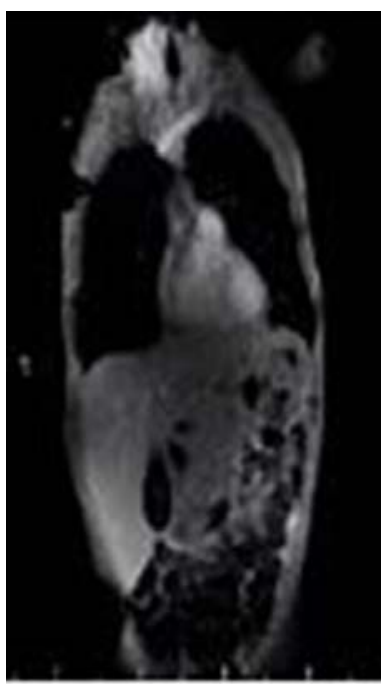

Figure 3. MRI images of the intrathoracic vascular abnormalities. 
A 60 year-old man with a 33 pack-year history of tobacco abuse, presented with difficulty speaking and voice change for several weeks. His review of systems was positive for fatigue, night sweats and weight loss. Physical exam of the oropharynx with flexible laryngoscopy demonstrated immobile bilateral true and false vocal cords fixed in the para-median position without laryngeal lesions. Concern for intra-thoracic process with recurrent laryngeal nerve involvement, a computed tomography (CT) of the chest and thoracic vessels demonstrated unusual appearing arteries with multiple penetrating atherosclerotic ulcers versus saccular aneurysms scattered throughout the aorta and its major branches (Figures 1 and 2). A magnetic resonance imaging (MRI) with contrast, demonstrated multiple foci of saccular outpouchings involving the arch vessels distal to their origins with the largest dilatation measuring $26 \times 25 \mathrm{~mm}$ in the case of proximal innominate (Figure 3). Although imaging lacked resolution, it was specialist opinion that patient likely had compression of recurrent laryngeal nerve leading to vocal cord dysfunction without significant airway compromise.

Atherosclerotic aneurysms of the great vessels are rare with evidence limited to case series $(1,2)$. Patient presentation varies dependent on the structures involved including embolic phenomenon from atherosclerosis. Surgical intervention with endovascular approach remains treatment of choice with good success rate.

Given our patient's poor nutritional status, multiple co-morbidities and diagnosis of large colonic mass, risks of surgery outweighed benefits of intervention and thus outpatient follow up was recommended.

Faraz Jaffer, MD and Don Leo Pepito, MD

Department of Internal Medicine

Banner-University Medical Center - South

Tucson, AZ

\section{References}

1. Cury M, Greenberg RK, Morales JP, Mohabbat W, Hernandez AV. Supra-aortic vessels aneurysms: diagnosis and prompt intervention. J Vasc Surg. 2009;49(1):410. [CrossRef] [PubMed]

2. Kasashima $\mathrm{F}$, Urayama $\mathrm{H}$, Ohtake $\mathrm{H}$, Watanabe $\mathrm{Y}$.Intrathoracic aneurysm of the innominate and right subclavian arteries: report of a case. Surg Today. 2001;31(1):51-4. [CrossRef] [PubMed] 